D.T. Wickramasinghe, L. Ferrario, and G.V. Bicknell, eds.

\title{
Simultaneous optical and EUVE Observations of the IP PQ Gem
}

\author{
Gavin Ramsay \\ Astronomical Institute, University of Utrecht, The Netherlands
}

Steve Howell

Dept Physics 8 Astronomy, Univ of Wyoming, Laramie, WY, USA

Martin Sirk

Center for EUV Astrophysics, University California, Berkley, CA, USA

Mark Cropper \& Stephen Potter

Mullard Space Science Lab, UCL, Surrey, England

Simon Rosen

Dept Physics \& Astronomy, Univ Leicester, Leicester, England

\section{Introduction}

The CV PQ Gem is thought by many to be the first truly 'Intermediate' Polar in that it displays characteristics typical of the synchronous and high magnetic field strength $(B \sim 10-230 \mathrm{MG})$ AM Her systems as well as those of the asynchronous (and much weaker magnetic field strength) Intermediate Polar systems. Here we present the results of quasi-simultaneous optical photopolarimetric and EUVE observations.

\section{Observations}

Optical Photopolarimetric I filter data were obtained between 1996 Jan 1517 using the $2.2 \mathrm{~m}$ telescope at ESO, Chile, and EUVE data $(70-170 \AA)$ were obtained between $1996 \mathrm{Jan} 13-19$ with $\sim 2$ days exposure in total. The EUVE data had a mean count rate of $0.07 \mathrm{cts} / \mathrm{sec}$.

\section{Results}

Figure 1 shows the EUVE light curve folded on the white dwarf spin ephemeris of Stavroyiannopoulos et al (submitted MNRAS), where $\phi=0.0$ defines the dip seen in X-rays which is due to the accretion stream obscuring the accretion region on the surface of the white dwarf. There is a time delay in the dip seen in the EUV compared with the X-ray dip $(40 \pm 17 \mathrm{sec})$. In the optical (also fig 1), 

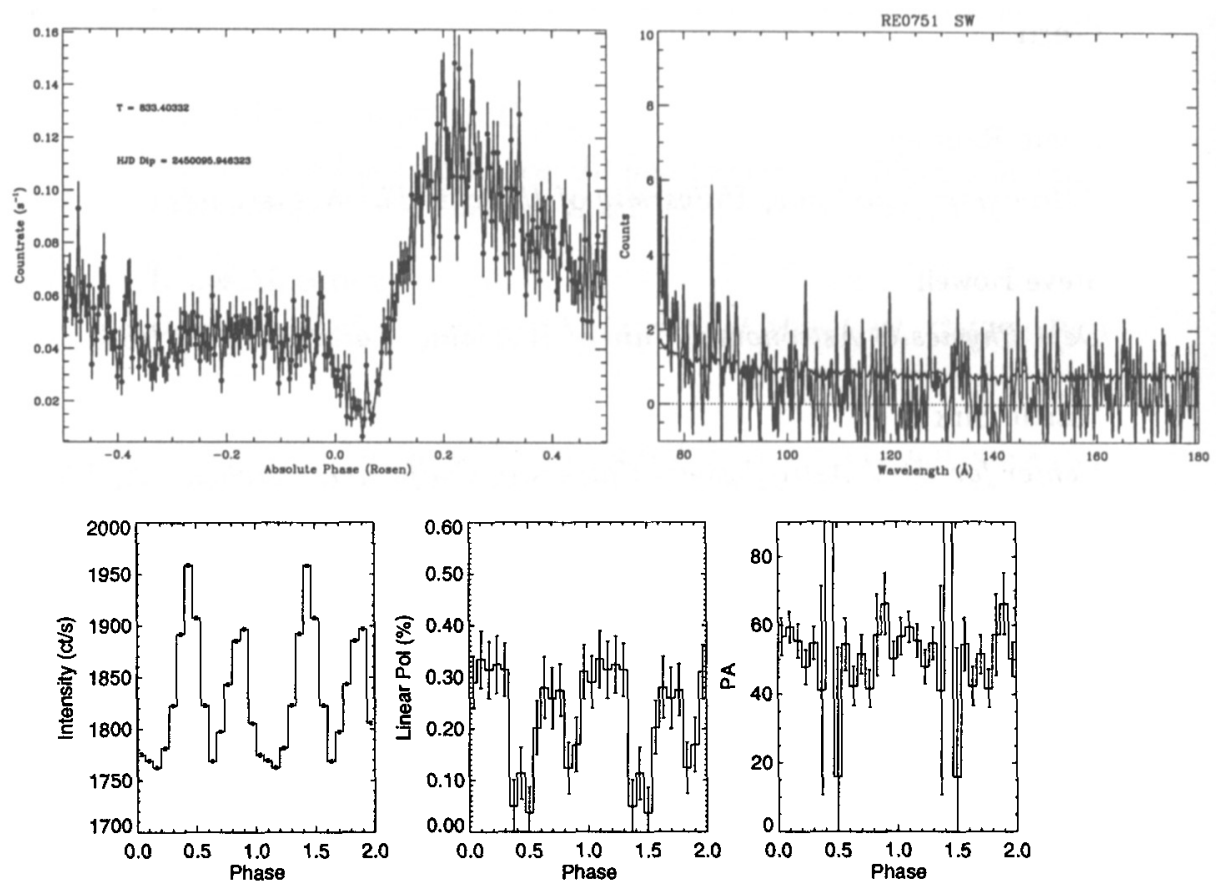

Figure 1. Top left: The integrated EUVE light curve; Top right: the integrated EUVE spectrum (the lower solid line is the $3 \sigma$ error estimate); lower panel: the optical $I$ band optical data, linear polarisation and its position angle (after subtraction of the interstellar polarisation). The light curves are folded on the spin ephemeris of Stavroyiannopoulos et al.

the dip is much broader than in X-rays or the EUV, and has a secondary dip half a cycle later which is probably due to cyclotron self-absorption.

The integrated EUVE spectrum is shown in fig 1 and has a weak to nonexistent EUV continuum with emission lines of highly ionised $\mathrm{Fe}, \mathrm{Ne}$ and possibly $\mathrm{Ni}$. These lines are consistent with a low temperature plasma of $T \sim 10^{7} \mathrm{~K}$. The low $\mathrm{S} / \mathrm{N}$ does not allow a detailed spectral fit.

The Stokes polarisation parameters $Q$ and $U$ are modulated on the spin period of the white dwarf $(13.9 \mathrm{~min})$. The spin folded and binned polarisation and intensity data are shown in fig 1 after the interstellar polarisation component has been subtracted $(p=0.2 \%)$. Moreover, a dip in the linearly polarisation flux is seen at $\phi \sim 0.3-0.5$ which is coincident to the main intensity peak. This is in contrast to the only other available linear polarisation data which showed a peak coincident with the main flux peak (Piirola et al 1993, ApJ, 410, L107). Using the relationship between interstellar polarisation and distance of Barrett (1996, PASP, 108, 412) we estimate the distance to PQ Gem $(220 \pm 60 \mathrm{pc})$. A more detailed paper is in preparation. 